\title{
Faraday rotation studies of the giant radio galaxy 3C33.1: An evolving naked-jet radio source?
}

\author{
Marcelo Vidalis ${ }^{1}$ and Everton Lüdke ${ }^{1}$ \\ ${ }^{1}$ Universidade Federal de Santa Maria, CCNE - Departamento de Física - LARIE, Campus \\ Universitário, 97150-900 Santa Maria RS, Brazil
}

\begin{abstract}
In this paper we present results of a detailed morphological and radio polarization study of the nearby radio galaxy 3C33.1 (4C72.01, 0106 +729, $z=0.18)$ made with the Very Large Array at 18 and $21 \mathrm{~cm}$. Recently, this galaxy has been re-classified within a sample of large core-dominated radio sources and it has an exceedingly high projected linear size of $\sim 450 \mathrm{kpc}$. The jet shows little Faraday rotation, with rotation measures $|R M|<20 \mathrm{rad} \mathrm{m}^{-2}$, whereas the hotspots exhibit higher values. The low rotation measures indicate that this radio source has a visible jet, which can be a naked jet or stripped from a dense environment. A spectral ageing analysis, based on syncrotron power losses arguments of the integrated spectra, suggest that the radio structure is not younger than 20 Myrs. Further observational properties of this radio galaxy are also discussed.
\end{abstract}

\section{Introduction}

Giant radio galaxies usually have projected linear sizes greater than $100 \mathrm{kpc}$ according to Lara et al. (1999) and Lara et al. (2001). They often show very low depolarization asymmetry in comparison with galactic-sized radio sources (Garrington, Leahy \& Conway (1988), Liu, Pooley \& Riley (1992) and amounts of integrated Faraday rotation about 10 times smaller than those found in the jet sides of GSRS. This observational result is interpreted in terms of the effects of a hot magnetized medium with a density profile decreasing radially with respect to the core position (Garrington \& Conway (1991)), and tangled intergalactic magnetic field lines. Lara et al. (2001) have put together a sample of large angular size radio galaxies and classified 3C33.1 among them.

In this paper, we report high angular resolution observations of the polarization structure of $3 \mathrm{C} 33.1$ based on available VLA observations at 18 and $21 \mathrm{~cm}$.

\section{The results}

The overall radio source morphology is that of a classical Fanaroff-Riley II. The radio jet and the lobes are resolved in our images. The jet shows multiples knots of enhanced radio continuum emission suggesting internal instabilities. A published $5 \mathrm{GHz}$ image shows these features at higher resolution. The source projected linar size is $256 \mathrm{Mpc}$ and the visible jet component is $26 \mathrm{kpc}$. If the jet is visible due to Doppler boosting, according to unification schemes ( Urry \& Padovani (1995)), we estimate a line-of-sight viewing angle of $\sim 24^{\circ}$.

The depolarization is about unitary in all regions of the radio source and hence the radio source does not show depolarization assymmetry. We can attribute this effect to the low intensity of intergalactic magnetic fields in comparison to those found in the interstellar medium of galactic-sized radio sources, or that the inhomogeneities in the 

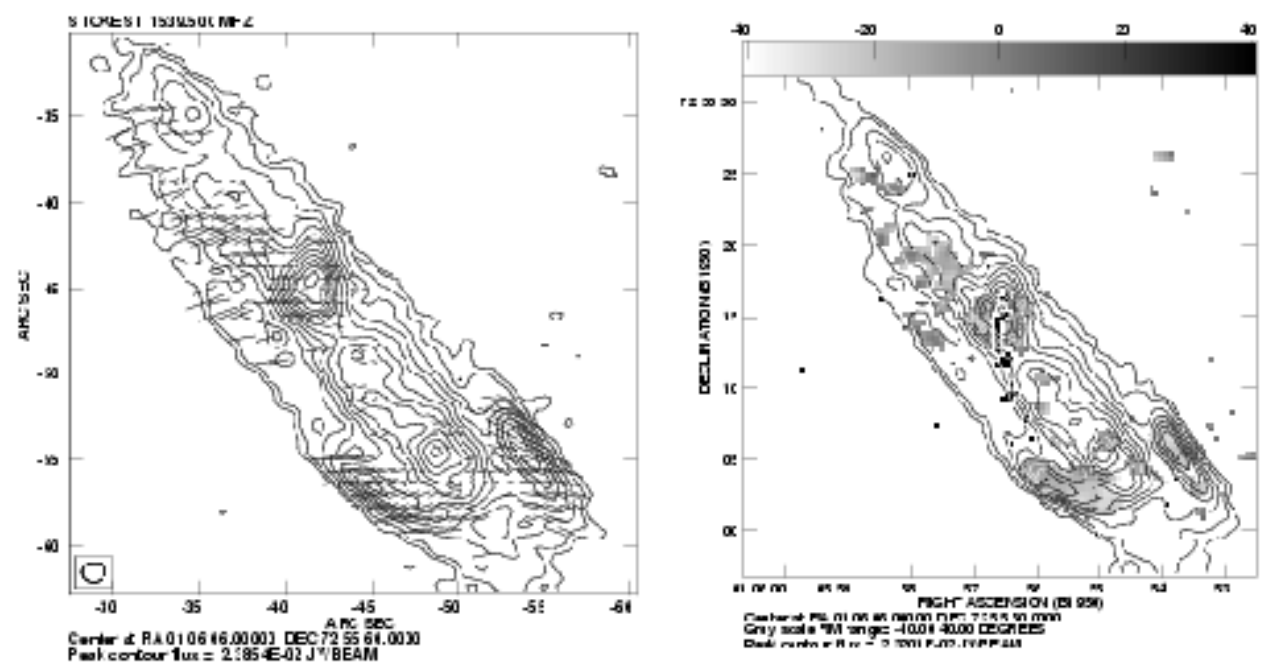

Figure 1. 21-cm polarized emission on the main components of 3C33.1. Vectors are scaled as 1 arcsec $=48 \%$ fractional polarization. Contours are multiples of two of the lowest contour of $95 \mu \mathrm{Jy}$ beam $^{-1}$. The rotation measures $\chi-\chi_{0}=R M \lambda^{2}$ (Laing (1985)) are given as greyscales. The restoring beam was $1.2^{\prime \prime}$, with a linear size of $\sim 4 \mathrm{kpc}$ at the source redshift $\left(\mathrm{H}_{0}=50 \mathrm{~km}\right.$ $\left.\mathrm{s}^{-1} \mathrm{Mpc}^{-1}, q_{0}=0.5\right)$.

Faraday depths to be resolved by the synthetic beam. The mean Faraday rotation measures were measured at the hotspots and turned out to be about $-20 \mathrm{rad} \mathrm{m}^{-2}$ for the counter-jet side and about $-18 \mathrm{rad} \mathrm{m}^{2}$ for the jet side. The jet itself has around $-18 \mathrm{rad}$ $\mathrm{m}^{2}$ over its visible extension. The differences are small in comparison with the errors and the rotation measures can be thought as the same throughout the source. This behaviour is typical of few naked-jet radio sources such as $3 \mathrm{C} 138$, in which rotation measures are small as if the plasma is stripped from a faraday-active screen (Akujor et al. (1993)).

Finally, we estimate a spectral cut-off frequency at about $8 \mathrm{GHz}$ for the hotspots. A simple spectral ageing analysis of the hotstpots suggests a dynamical age of $~ 60$ Myrs, which is consistent with a single free-expanding radio source against a homogeneous IGM with a hotspot velocity of $0.06 \mathrm{c}$. We also interpret our results as the radio component is expanding through the intergalactic medium with an expected mean particle density less than $6 \times 10^{-2} \mathrm{~cm}^{-3}$ at the hotspots and equipartition magnetic fields in the range $20-40 \mu \mathrm{G}$.

\section{Acknowledgements}

The Very Large Array national facility is operated by the NRAO on behalf of NSF.

\section{References}

Akujor, C. E., et al. 1993, A\&A, 274, 752

Garrington, S. T., \& Conway, R. G. 1991, MNRAS, 250, 198

Garrington, S. T., Leahy, J. P., Conway, R. G., \& Laing, R. A. 1988, Nature, 331, 147

Laing, R.A. 1985, in Physics of Energy Transport in Extragalactic Radio Sources, (eds. A. Bridle, Eilek, J.), NRAO publication

Lara, L., Márquez, I., \& Cotton, W. D., et al. 1999, A\&A, 348, 699

Lara, L., et al. 2001, A\&A, 370, 409

Liu, R., Pooley, G., \& Riley, J. M. 1992, MNRAS, 257, 545

Scheuer, P. A. G., \& Readhead, A. C. S. 1979, Nature, 277, 182

Urry, C. M., \& Padovani, P. 1995, PASP, 107, 803 